\title{
Neue und relevante Studien
}

\author{
Alain Farron ${ }^{\mathrm{a}}$, Roland M. Biedert ${ }^{\mathrm{b}}$ \\ a Prof. Dr. med., Präsident swiss orthopaedics; ${ }^{b}$ Prof. Dr. med., Präsident Expertengruppe Knie swiss orthopaedics
}

Das Fachgremium des SMB hat der Beurteilung der Expertengruppe Knie (EGK) swiss orthopaedics [1] in ihrer Replik widersprochen und hält dabei an den Empfehlungen aus den Jahren 2009 und 2013 fest [2]. Leider hat dies keine neuen Aspekte oder Klärungen ergeben. Das SMB geht in seiner Replik nur kurz und generell formuliert auf einzelne Punkte der Beurteilung der Expertengruppe Knie swiss orthopaedics ein. Abschliessend hält das SMB fest, dass keinerlei neue Daten, Informationen oder Argumente zu erkennen sind, die für die Empfehlungen im Fachbericht des SMB relevant wären. Die Replik des SMB erfordert deshalb eine zweite Stellungnahme der Expertengruppe Knie swiss orthopaedics.

\section{Kompetenz des Fachgremiums SMB}

Das SMB bestätigt, dass das Gremium selbst nicht über Fachspezialisten zu diesem Thema verfügt. Es wurden deshalb drei themenspezifische, externe Kollegen befragt. Es ist für die Expertengruppe Knie (EGK) unerklärlich, wie in wenigen Stunden ein solch komplexes und relevantes Thema mit dieser Tragweite abschliessend von Kollegen, die nicht der EGK angehören, abgehandelt und der SMB-Fachbericht anhand einer solchen kurzen Befragung von SMBNichtspezialisten verfasst werden kann.

\section{Neue relevante Literatur}

Die Expertengruppe Knie swiss orthopaedics hat auch nach der Replik des SMB ab 2013 unter spezieller Berücksichtigung der vom SMB häufig erwähnten Kosten-Nutzwert-Analyse eine kontinuierliche Literaturrecherche fortgesetzt und zwei neue und relevante Studien $\mathrm{zu}$ dieser Thematik gefunden.

\section{Eine ausführlichere Stellungnahme findet sich unter www.saez.ch $\rightarrow$ Aktuelle Nummer oder $\rightarrow$ Archiv $\rightarrow 2015 \rightarrow 40$}

Eine Antwort des SMB findet sich im Anschluss.

\section{Studie 1: Mather RC, Hettrich CM, Dunn WR et al.} Cost-Effectiveness Analysis of Early Reconstruction Versus Rehabilitation and Delayed Reconstruction for Anterior Cruciate Ligament Tears. Am J Sports Med. 2014;42:1583-91)

In Anlehnung an die Studien von Frobell et al. führten die Autoren in einer eigenen Studie eine Kosten-Nutz-
wert-Analyse durch. Sie verglichen dabei die vordere Kreuzbandrekonstruktion (VKB-R) mit der gezielten Rehabilitation (für 24 Wochen) und wahlweiser späterer VKB-R und analysierten zwei hauptsächliche Aspekte:

1. Vergleich der qualitätskorrigierten Lebensjahre zwischen früher VKB-R ( $<10$ Wochen) und wahlweise späterer VKB-R. 2. Vergleich der direkten Kosten dieser zwei Gruppen von einem gesellschaftlichen Gesichtspunkt. Spezifische Resultate zeigten: a) 71\% der Patienten aus der Gruppe mit gezielter Rehabilitation wiesen nach 2 Jahren eine Instabilität auf; b) 39\% unterzogen sich einer sekundären VKB-R innerhalb von 2 Jahren, weitere 10\% zwischen 2-6 Jahren (total 49\%). Das Gesamtresultat ergab, dass eine frühe VKB-R sowohl kostengünstiger als auch effektiver ist als eine primäre Rehabilitation und wahlweise sekundäre VKB-R und dass für eine optimale medizinische Versorgung die frühe VKB-R zu bevorzugen ist.

Kurz- und langfristig führte die VKB-R zu besserer Lebensqualität und geringeren Kosten verglichen mit Rehabilitation.

Studie 2: Mather RC, Koenig L, Kocher MS et al. Societal and economic impact of anterior cruciate ligament tears. J Bone Joint Surg Am. 2013;95:1751-9)

Die Autoren führten eine Kosten-Nutzwert-Analyse der frühen VKB-R ( $<10$ Wochen) durch und verglichen sie mit einer gezielten Rehabilitation. Kurz- bis mittelfristige Resultate (bis 6 Jahre) und Langzeitresultate (lebenslang) wurden analysiert. Zur Beurteilung der Wirksamkeit der Behandlung wurden die qualitätskorrigierten Lebensjahre sowie die Kosten in US-Dollars verwendet. Die indirekten Kosten wurden dabei definiert als Lohnverlust, Produktivitätsverlust und Erwerbsunfähigkeit als Folge einer persistierenden Instabilität. Diese indirekten Kosten waren hauptsächlich auf die Instabilität und weniger auf die Entwicklung einer Arthrose zurückzuführen. Die kurz- bis mittelfristigen Resultate zeigten, dass die VKB-R sowohl günstiger ( "a cost reduction of \$ 4503») als auch effektiver ("a QALY gain of 0.18») waren verglichen mit der gezielten Rehabilitation. Langfristig betrugen die lebenslangen Kos- 
ten für Patienten mit VKB-R USD 38121 verglichen mit USD 88538 für Rehabilitation. VKB-R verursachte demnach USD 50417 weniger Kosten mit einem Qualitätsgewinn von 0,72 verglichen mit Rehabilitation.

Zusammenfassend führte sowohl kurz- wie langfristig die VKB-R zu besserer Lebensqualität und geringeren Kosten verglichen mit gezielter Rehabilitation.

\section{Statistik der SUVA}

Die SUVA-Rohdaten 1990-2011 (Rate OP-VKB, SUVA Versicherte) zeigen zudem, dass heute nur die Hälfte der VKB-Rupturen operiert werden. Anfang 2000 waren es noch über 80\%, 2011 noch 50\%. Diese Abnahme über die letzten zehn Jahre ist auf die allgemeingültige Schweizer best practice zurückzuführen.

swiss orthopaedics soll als zuständige Fachgesellschaft zur Beratung des SMB-Gremiums direkt involviert werden.

Wesentlich ist dabei, dass die deutliche Abnahme schon vor den Empfehlungen des SMB durch die operierenden Ärzte umgesetzt wurde und bestätigt deren verantwortungsvolle Indikationsstellung.

Korrespondenz: Prof. Dr. med. Alain Farron swiss orthopaedics Chemin de la Bovarde 19 CH-1091 Grandvaux

\section{Wichtigste Fakten und Erkenntnisse}

- Bei zukünftigen Beurteilungen von komplexen Fragestellungen soll swiss orthopaedics als zuständige
Fachgesellschaft zur Beratung des SMB-Gremiums direkt involviert werden.

- Die neuesten Studien in hochklassierten Fachzeitschriften zeigen eindeutig, dass eine frühe VKB-R einen besseren Kosten-Nutzwert als die gezielte Rehabilitation mit möglicher sekundärer VKB-R hat. Dies ist darauf zurückzuführen, dass bei diesen Studien nicht nur die direkten, unmittelbaren Kosten (Operation versus Rehabilitation), sondern auch die indirekten, langfristigen Kosten (Lohnverlust, Produktivitätsverlust und Erwerbsunfähigkeit als Folge einer persistierenden Instabilität) in die Analyse miteinbezogen wurden. Geht es dem SMB wirklich um den häufig aufgeführten Kosten-Nutzwert, dann ist dies die klare Antwort.

- Die neue Statistik der SUVA bestätigt, dass in den letzten 10 Jahren viel weniger primäre VKB-R durchgeführt wurden. Dies, weil die Indikationen der Fachspezialisten schon lange viel zurückhaltender gestellt wurden.

Die EGK swiss orthopaedics weist deshalb die Replik des SMB zurück.

\section{Literatur}

1 Christen B, Biedert RM. Review des SMB-Fachberichts «Ruptur des vorderen Kreuzbandes». Schweiz Ärztezeitung. 2014;95(29/30):1089-91.

2 Metzger U, Bohnenblust H. Replik des Swiss Medical Board zur Stellungnahme von swiss orthopaedics. Schweiz Ärztezeitung. 2014;95(29/30):1092

\section{ENVOYEZ VOS PROJETS AVANT LE 8 JANVIER 2016}

Le Swiss Quality Award récompense les meilleures innovations en matière de qualité dans le système de santé. Participez et enregistrez votre projet sur le site www.swissqualityaward.ch. 